\title{
Twitter y la teoría de la Agenda-Setting: mensajes de la opinión pública digital
}

\author{
Raquel RUBio GARCÍA \\ Universidad Carlos III de Madrid \\ raquelrubiog@gmail.com
}

Recibido: 16 de septiembre de 2013

Aceptado: 10 de marzo de 2014

\begin{abstract}
Resumen
El presente artículo pretende comprobar si la teoría de la Agenda-Setting mantiene su vigencia dentro del creciente entorno digital; concretamente dentro del contexto de los social media como categoría en la que se engloba la red social Twitter. Se parte de la consideración de que Twitter se ha constituido como un reflejo de la agenda pública. Por ello, resulta esencial analizar cuáles son las cuestiones que más interesan a los usuarios españoles, para determinar si los medios de comunicación tradicionales marcan los temas más comentados por los usuarios de Twitter España, entendidos estos como opinión pública. Esta investigación ha encontrado que existe una fuerte correspondencia entre la agenda de los medios y la agenda pública reflejada en Twitter.
\end{abstract}

Palabras clave: Agenda-Setting, Twitter, agenda pública, agenda mediática, medios sociales.

\section{Twitter and the Agenda-Setting theory: messages from the digital public opinion}

\begin{abstract}
This paper aims to test whether or not the Agenda-Setting theory is still applicable in the growing digital environment; particularly within the context of social media as a category where the social network Twitter is included. The research starts from the consideration that Twitter has become a reflection of the public agenda. Thus, analyze which issues matter most to Spanish users is essential to determine if traditional media establishes the topics most discussed by users of Twitter Spain, understanding them as public opinion. This research has found that there is a strong correspondence between the media agenda and the public agenda shown in Twitter.
\end{abstract}

Keywords: Agenda-Setting, Twitter, public agenda, media agenda, social media.

\section{Referencia normalizada}

RUBIO GARCÍA, Raquel (2014): “Twitter y la teoría de la Agenda-Setting: mensajes de la opinión pública digital”. Estudios sobre el Mensaje Periodístico. Vol. 20, Núm. 1 (enero-junio), págs.: 249-264. Madrid, Servicio de Publicaciones de la Universidad Complutense.

Sumario: 1. Introducción. 2. Fuentes y metodología. 3. Twitter: la agenda pública o del público. 4. El País y El Mundo: la agenda mediática y su correlación con la agenda pública. 5. Conclusiones. 6. Referencias bibliográficas.

\section{Introducción}

Como es de sobra conocido, la teoría de la Agenda-Setting fue formalmente desarrollada en 1972 por el sociólogo estadounidense Maxwell McCombs junto a Donald Shaw a partir de los trabajos previos del periodista Walter Lippmann (1922). Esta postula que los medios de comunicación, al seleccionar los temas que incluyen y omiten en su agenda, ejercen gran influencia sobre el público y sobre su capacidad para opinar y debatir los asuntos públicos, ya que determinan los temas de interés informativo, su importancia, y el espacio que se dedica a cada uno. 
Sobre la evolución de dicha Teoría en los nuevos entornos digitales y los cambios en los paradigmas de comunicación, el propio McCombs resaltó en 2004' que "la teoría de laAgenda-Setting es un complejo mapa intelectual que aún se halla en proceso de evolución" (McCombs, 2006: 20). Un año después reconocía las nuevas formas de comunicación digitales y sus posibilidades de estudio, pero sostuvo que Internet y los medios sociales no hacen sino corroborar los principios de la Teoría, en los que son los medios los que marcan la agenda temática de la opinión pública (McCombs, 2005).

McCombs no abordó el nuevo rol de las redes sociales, pero centró su atención en los blogs, sobre los que aseguraba, eran "subsidiarios de los medios tradicionales: versiones online de los periódicos, revistas, cadenas de televisión y canales de noticias por cable" (McCombs, 2005: 545). Estudios como el del Pew Research Centre (2010) y K. Bruder (2008) reafirmaron esta visión, mientras que otros como el de N. Rostovtseva (2009) y S. Meraz (2009) cuestionaron la influencia social de los medios tradicionales y su capacidad de establecer la agenda de los ciudadanos en la blogosfera.

En las últimas dos décadas las investigaciones y estudios enmarcados dentro del campo de la teoría de la Agenda-Setting se han centrado en los medios de comunicación digitales como nuevos medios capaces de influir en la opinión pública de forma similar a como lo hacen los tradicionales. Sin embargo, pocos se han centrado en la red social Twitter.

Quizá uno de los más relevantes sea el ya citado estudio del Pew Research Center en 2010. Este trabajo, que compara los contenidos de blogs, Youtube y Twitter entre ellos y con los ofrecidos por los medios de comunicación, encontró que la mitad (50\%) de los enlaces llevaban a medios existentes, un porcentaje muy bajo si se compara con el 99\% registrado en blogs. La conclusión general fue que "las historias y cuestiones más importantes en los medios sociales difieren sustancialmente de aquellas que lideran la prensa" (Pew Research Center, 2010: 1).

Sin embargo, Meghan Krane publicó en 2010 una tesis centrada en el análisis de los contenidos difundidos en Twitter por tres medios - The New York Times, CNN y $N P R$-. El estudio concluyó que existe una relación directa entre los contenidos divulgados por los medios y los temas que con mayor frecuencia son abordados por los usuarios (Krane, 2010).

Dentro del medio digital, otros trabajos han tratado las cuestiones de direccionalidad de la información y procedencia de las fuentes. En este sentido, en 2011 José Luis Orihuela publicó en uno de los blogs de $A B C$ el post "Los trending topics como el nuevo Agenda-Setting", en el que consideraba que las plataformas web de filtrado social de información como Twitter habían revertido el proceso de influencia llevándolo desde el público hacia los medios.

La mayoría de estas aportaciones han tratado de contemplar la evolución de la teoría de la Agenda-Setting en el entorno mediático desencadenado por Internet a tra-

1 Año de publicación del libro en lengua inglesa.

2 Publicado en el blog de ABC Digital Media Weblog el 28 de febrero de 2011: http://www.abc.es/blogs/jose-luis-orihuela/public/post/los-trending-topics-como-el-nuevoagenda-setting-8123.asp [fecha de consulta: 15 de marzo de 2013]. 
vés del nuevo papel que están jugando plataformas como los blogs o las páginas web de los medios tradicionales. Sin embargo, sus principios no han sido especialmente explorados dentro de la red social Twitter, el servicio de microblogging que en 2012 contaba con 517 millones de perfiles en todo el mundo ${ }^{3}$.

Por esta razón consideramos que resulta de especial utilidad conocer qué interesa a los usuarios de Twitter y cuáles son los temas más comentados en la red social, puesto que estos son los componentes de la agenda del público. Además, averiguar si los temas tratados por los internautas coinciden con los abordados por los medios de comunicación tradicionales no sólo ayudará a dilucidar el sentido en el que fluye la información, sino que también permitirá descubrir hasta qué punto la teoría de la Agenda-Setting puede constituir un referente en estudios sobre comunicación en el actual panorama mediático digital.

\section{Metodología}

Teniendo en cuenta la enorme amplitud de nuestro objeto de estudio principal (Twitter), la metodología seguida por esta investigación se ha basado principalmente en la técnica del análisis de contenido (Berelson, 1952; Krippendorff, 1990; Wimmer et al., 2006), adaptada al entorno de Twitter y desarrollando dos análisis distintos. El primero ha permitido analizar los contenidos más compartidos en España durante el mes de marzo de 2013 a través de los trending topics o temas de moda más comentados por los usuarios. El segundo ha sido el estudio de dichos temas de moda en las versiones digitales de El País y El Mundo, los dos periódicos de información general más leídos en España, de acuerdo con los datos publicados en el EGM de febrero a noviembre de $2012^{4}$.

Para llevar a cabo el primer análisis hemos utilizado como herramienta principal Tweettrendings ${ }^{5}$, una aplicación online que permite examinar los trending topics de España. Gracias a su base de datos y su buscador, se han contabilizado los 3.613 trending topics de marzo y clasificado en función de su temática en trece categorías que se han englobado en dos bloques.

El primero de ellos está formado por aquellas categorías que se corresponden con las secciones en las que suelen dividir la información los periódicos: 'Política', 'Internacional', 'Deportes', 'Economía', 'Religión' y 'Sociedad', y sus respectivas variables. El segundo enmarcaría aquellas que no suelen ser registradas en los periódicos porque son muy específicas o porque pueden ser consideradas como originarias de Twitter. Este bloque está compuesto por: 'Música', 'Personajes'-para los trending topics que aluden a personas no pertenecientes al mundo de la política, los deportes o

3 Cifras obtenidas del informe de la compañía de análisis francesa Semiocast. http://semiocast.com/publications/2012_07_30_Twitter_reaches_half_a_billion_accounts_140m_in_the US [fecha de consulta: 25 de febrero de 2013 ].

${ }^{4} . \overline{\mathrm{h}} \mathrm{ttp}: / /$ www.aimc.es/spip.php?action=acceder_document\&arg=2222\&cle=a2fbe573ed3cbe3 a39da5de72f1a42dd6d1582d5\&file=pdf\%2Fresumegm312.pdf [fecha de consulta: 5 de febrero de 2013].

${ }^{5}$ http://www.tweettrendings.com/ [fecha de consulta: 16 de febrero de 2013]. 
la música-, 'Concursos', 'Programas' -referente a la programación televisiva y radiofónica-, 'Participación' -para los temas que buscan la participación expresa de los usuarios para compartir opiniones, intereses, experiencias...-, 'Happy/feliz' -relativa a los trending topics que comienzan con uno de estos dos términos- y 'General' -para aquellos que engloban temáticas muy variadas sin un patrón concreto-.

Esta clasificación permite no sólo una comparación horizontal entre los temas más populares del mismo día, sino también una comparación vertical entre los diferentes días del mes.

En el segundo análisis se han contemplado solamente los trending topics englobados en el primer bloque, de los que se ha realizado un seguimiento diario a través de las barras de búsqueda de El País y El Mundo con el objetivo de contabilizar el número de ellos que fueron recogidos por los diarios, y el número de noticias dedicadas a cada temática.

Paralelamente a la metodología cuantitativa, se ha utilizado una cualitativa en su técnica de la entrevista en profundidad (Taylor et al., 1984; Ruiz Olabuénaga, 2012; 1984; KVALE, 2011). Las entrevistas con diversos expertos han aportado a este trabajo un conocimiento extra no sólo sobre los estamentos que componen el paradigma de la Agenda-Setting, sino sobre sus correlaciones en los nuevos entornos digitales ${ }^{6}$.

\section{Twitter: la agenda pública o del público}

La agenda pública ha adquirido un nuevo reflejo con el auge de Internet y los medios y redes sociales. Los primeros estudios de McCombs buscaron una comprensión profunda de los temas abordados por los ciudadanos en el discurso público a través de métodos como los cuestionarios. Sin embargo, el desarrollo de las tecnologías ha propiciado que la opinión pública disponga de un nuevo espacio de discusión: el digital. En este sentido, entre la multitud de plataformas que existen -foros, blogs...- las redes sociales, y más concretamente Twitter, han desempeñado un papel fundamental como altavoz y reflejo de la agenda del público.

Desde el punto de vista de Manuel de Ramón, "las redes sociales, Twitter en este momento y otras en cinco años porque al final Twitter acabará siendo superado como parece que ha ocurrido con los blogs, nos aproximan a la idea de qué es lo que inquieta a los usuarios, a los ciudadanos. Nos aproxima a los temas sobre los que escriben, sobre los que comentan, discuten..."”.

Candón Mena también recogió esta idea asegurando que "las redes sociales son razonadas como un medio más permeable al influjo de las agendas ciudadanas hasta el punto de considerarse un medio de "autocomunicación de masas" que, por su hori-

6 Con este objetivo han sido entrevistados Raquel Rodríguez, autora del libro "Teoría de la Agenda-Setting: aplicación a la enseñanza universitaria” (Rodríguez, 2004), Fermín Bouza, experto en Agenda-Setting y comunicación política (Bouza et al., 2004) y Manuel de Ramón Carrión, profesor de la Universidad Complutense de Madrid y experto en periodismo especializado.

7 Declaraciones de M. de Ramón en entrevista concedida a la autora el día 26 de junio de 2013. 
zontalidad e interactividad, constituiría un reflejo más fidedigno de la verdadera opinión pública, en contraste con la opinión publicada en los medios tradicionales".

Por ello, partiendo de la consideración de que los temas más comentados en España por los usuarios de Twitter son una muestra real de las temáticas que constituyen la agenda pública, es interesante observar la distribución de los temas en las distintas categorías.

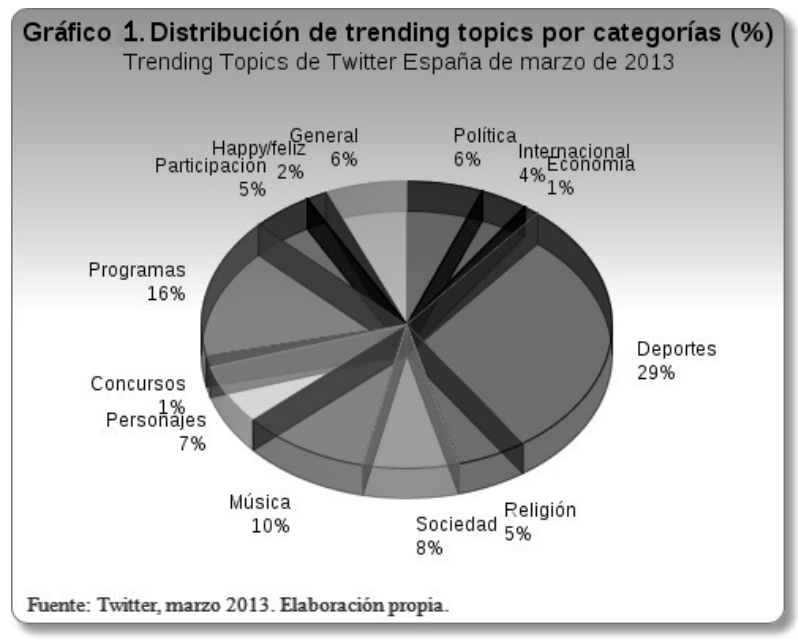

El 29\% de ellos (1.065 trending topics) hicieron referencia a cuestiones relacionadas con el mundo del deporte, categoría que casi duplica a la siguiente más numerosa -'Programas'-, que con 569 temas (16\% del total) se coloca en segunda posición. Por detrás se encuentran 'Música' y 'Sociedad', donde se han englobado el 10\% y 8\% respectivamente.

Por su parte, 'Concursos' (44), 'Economía' (45) y 'Happy/Feliz' (59), resultaron ser las que menor número de temas de moda acumularon. De acuerdo con la división explicada anteriormente, se puede asegurar que la mayoría de los trending topics analizados pertenecen al grupo de categorías típicamente recogidas por los periódicos, pues suponen el $53 \%$ del total.

De los resultados se desprende que los usuarios de Twitter España han manifestado una clara inclinación hacia los contenidos relacionados con el deporte y, aunque otros temas de actualidad política, económica o internacional no son tan numerosos, su inclusión como categoría muestra una tendencia de la opinión pública a abordar cuestiones relativas a acontecimientos actuales tanto de ámbito nacional como internacional.

No obstante, el porcentaje restante de trending topics (47\%) es suficientemente elevado como para advertir un incipiente interés de los usuarios por cuestiones rela-

${ }^{8}$ Candón Mena, José Luis. "La batalla de las agendas: De las redes sociales a la agenda mediática, política y electoral". Artículo inédito. Agradecemos a su autor que nos haya facilitado una copia para esta investigación. 
cionadas con la participación y el entretenimiento individual o colectivo. Además, las cifras obtenidas por "Concursos", "Participación", y "Programas" resumen la consideración de Twitter como una red social de alto nivel de implicación y participación.

En lo que respecta a las tendencias que sobresalen dentro de cada categoría señalada, hemos de destacar que en política se han registrado 217 trending topics, entre las que han destacado las cuestiones relacionados con la corrupción política, que han acumulado el 59\% de ellos; en contraposición a otros como los asuntos de la realeza española, que supusieron solamente el $8.8 \%$. La evolución a lo largo del mes ha sido sostenida pues no se ha registrado ningún día en el que los usuarios no participaran en temas relacionados con la política española.

Referente a las cuestiones internacionales, estas se han movido entre las menos populares, ya que los 134 temas que la componen sólo equivalen al 4\% del total. Las diferencias numéricas entre las temáticas son muy significativas. Los usuarios no participaron en conversaciones sobre países de Europa que no forman parte de la Unión Europea ni sobre países africanos. Por el contrario, los asuntos internacionales sobre los que han comentado están principalmente asociados a acontecimientos que afectan a la Unión Europea y a América del Sur. Las razones que pueden explicar este comportamiento pueden deberse a la cercanía geográfica y comunitaria por un lado, y lingüística por otro.

Los temas sobre la UE han sido los más numerosos (55): el 41\% de los internacionales, aunque en el cómputo global sólo suponen el 1.57\%. Además, gran parte de ese porcentaje se asocia a un país concreto: Chipre y su posible rescate por parte de la Unión Europea, un asunto que en marzo de 2013 vivió momentos transcendentales. En ese mes, se dieron a conocer las condiciones impuestas por la UE para el plan de rescate, el Plan B del gobierno chipriota, las limitaciones de retirada de capital impuestas por los bancos, las críticas del presidente chipriota a las autoridades de la eurozona, etcétera. El caso de Chipre y su reflejo en Twitter a través hashtags críticos como \#TroikaGameOver o \#HandsOffCyprus, nos sirven para demostrar, una vez más, la actualidad de los temas debatidos en Twitter.

Por su parte, los temas concernientes a América del Sur han logrado alcanzar un $1.38 \%$ en el recuento general. Los estados sudamericanos han mostrado una tendencia muy extrema en la concentración en torno a acontecimientos puntuales. Cerca del $89 \%$ de los temas estuvieron relacionados con Venezuela, la muerte de su presidente Hugo Chávez y el papel desempeñado por su sucesor Nicolás Maduro.

Algo similar ocurre con los asuntos relativos al continente asiático. Estos, a la vez que muestran un interés de los usuarios proporcionalmente bajo con respecto a otras zonas, son el reflejo de un único acontecimiento: el conflicto entre Corea del Norte y del Sur.

En lo que se refiere a la atención prestada por los usuarios de Twitter al ámbito deportivo, destaca el hecho de que es el tema que mayor interés despierta con 1.065 (57\% del total), y uno de los que mayores cambios han experimentado. La evolución a lo largo del mes se ha caracterizado por pronunciados ascensos y descensos. Este fenómeno se explica porque los internautas sólo generan contenidos sobre deportes cuando se produce algún encuentro deportivo, pero los días en los que no se retrans- 
mite ninguna actividad deportiva, sus contribuciones a la red disminuyen drásticamente y la prueba más clara se encuentra en la Fórnula1.

El fútbol ha sido el deporte más comentado en Twitter, pues ha ocupado el 20.3\% de los temas en el recuento general. Se trata de una cifra elevada si la comparamos con el resto de variables pues la siguiente más numerosa ha sido 'Personajes Televisivos', que se encuentra 15 puntos porcentuales por debajo.

También es reseñable que el baloncesto es el deporte más frecuentemente referenciado para encuentros entre equipos no españoles y que los temas vinculados a la Fórmula1 sólo fueron anotados durante los fines de semana, cuando tienen lugar las carreras del Mundial de F1.

Entre los intereses de los usuarios existe cierta tendencia hacia el deporte nacional y los deportistas españoles, jueguen estos en equipos españoles o internacionales. Estos resultados resaltan una nueva realidad y es que los tuiteros comentan en directo los acontecimientos deportivos que están viendo, permitiendo a aquellos que no tienen acceso a ellos su seguimiento vía Twitter.

En lo relativo a temas económicos es destacable que estos se han situado entre los menos comentados en Twitter. Los 45 temas de moda que hacen referencia a la economía española (reformas, bolsa, finanzas, mercado, empresas, etc.), sólo han supuesto un $1.25 \%$ del total. A pesar de que los ciudadanos españoles están muy preocupados por la crisis económica de España, esta preocupación encontró muy poco reflejo en Twitter, al menos durante marzo.

Especial mención merecen los asuntos que conciernen a las reformas económicas del Gobierno Español. Estos han sido únicamente dos: IVA y \#Austericio. Este último fue creado con anterioridad, pero el día ocho consiguió llegar a ser trending topic nacional. Por su parte, las cuestiones relacionadas con los bancos se han concentrado en torno a un número bastante reducido de entidades, a saber, La Caixa, el Banco de España y Bankia. De igual forma, los temas relacionados con la Bolsa giraron únicamente en torno a los bonos del Tesoro, al IPC -Índice de Precios al Consumidor, a la CNMV -Comisión Nacional del Mercado de Valores y al índice Dow Jones.

Concerniente a asuntos religiosos se han contabilizado 198 trending topics que, además de suponer un 5\% del total, se reparten de manera bastante igualitaria. Los resultados permiten concluir que los internautas no sólo siguen acontecimientos deportivos a través de Twitter, sino todo tipo de eventos, incluso religiosos y de carácter internacional. Concretamente en marzo, la categoría 'Religión' registró muy pocos temas no referentes al Cónclave para elegir al nuevo Papa o a la Semana Santa.

En alusión a 'Sociedad' es necesario aclarar que engloba temáticas muy distintas que han acumulado 272 trending topics. La mayor parte (61.8\%) han referenciado sucesos puntuales, mientras que otras cuestiones como los desahucios en España o las manifestaciones no son excesivamente comentadas por los usuarios.

En lo que respecta a materia musical, 361 referencias han colocado la música en el tercer puesto de la lista de los temas más populares, superando el $10 \%$ del total. Los resultados exponen un interés de los usuarios por cuestiones musicales centradas tanto en la figura de los artistas como en sus espectáculos. Las personalidades musicales fueron el centro de muchas conversaciones, pero también lo fueron los perso- 
najes televisivos, radiofónicos, etcétera, que lograron 245 trending topics distribuidos de manera desigual con un predominio absoluto de los relacionados con la televisión, (75\% del total).

Estos datos manifiestan nuevamente la creciente tendencia entre los usuarios de comentar en las redes, y sobre todo en Twitter, los contenidos que aparecen en los medios de comunicación tradicionales, sobre todo cuando se trata de programas televisivos de entretenimiento.

Sin embargo, esta tendencia se invierte cuando se trata de concursos en los medios. En general, los usuarios no están muy interesados en este tipo de interacción con los medios, ya que ha sido la categoría que ha registrado menor número de temas $(1.22 \%)$. Esta inactividad sorprende si tenemos en cuenta que las actividades de ver televisión o escuchar radio son altamente compatibles con el manejo de Twitter, al tratarse de dispositivos distintos.

De hecho, los datos referentes a programas, tanto de radio como de televisión, sí hacen pensar que los usuarios suelen utilizar la red social mientras ven contenidos televisivos o escuchan la radio. Para empezar, las cuestiones relativas a programas se han situado como las segundas más comentadas en Twitter, pues sus 569 temas de moda han supuesto el $16 \%$ del total analizado.

Como ocurrió con los personajes, de nuevo la televisión acapara una inmensa mayoría de los temas de moda: los programas televisivos han supuesto el $75 \%$ de este grupo.

Otro tipo de interactividad ha sido la recogida en 'Participación', que con 172 referencias ha logrado alcanzar un 5\% del total. Los temas relacionados con la participación dentro de la comunidad de usuarios de Twitter corroboran que están muy interesados en las distintas formas de participación que ofrece la red social.

Por su parte, la categoría 'Happy/Feliz', ha reunido 59 temas. Es una cifra reducida ya que sólo supone un $1.63 \%$ del total, pero es necesario recordar que en ella se engloban solamente los temas de moda que comienzan por el término feliz o happy.

Finalmente, se han contabilizado 232 temas generales que sólo suponen el 6\% del total, lo que indica que la inmensa mayoría de los trending topics han sido satisfactoriamente englobados en las restantes categorías. Cabe destacar que aproximadamente el $70 \%$ de los temas generales se han configurado a partir de nombres de ciudades, países y continentes, entre los que los términos España y Madrid han sido los dos más utilizados.

\section{El País y El Mundo: la agenda mediática y su correlación con la agenda pública} Una vez conocidos los temas que componen la agenda pública, y teniendo presente el objetivo que aquí se persigue de buscar la correlación entre esta y la agenda mediática; a continuación se exponen los resultados observados en el análisis de los temas informativos recogidos en Twitter, en El País y El Mundo, ambos en sus versiones digitales.

En términos muy generales se puede concluir que ambos diarios han registrado la mayoría de las cuestiones a las que se refieren los trending topics analizados, pues en ninguna de las variables el número de temas que El País y El Mundo no han reseñado suponen más del $45 \%$. 
Sin embargo, es cierto que se han observado variaciones importantes entre las temáticas. Mientras que la inmensa mayoría de las cuestiones internacionales y deportivas han encontrado su reflejo en la prensa, aquellas consideradas como parte de la categoría 'Sociedad', no han contado en estos diarios con el mismo peso otorgado por los usuarios en Twitter.
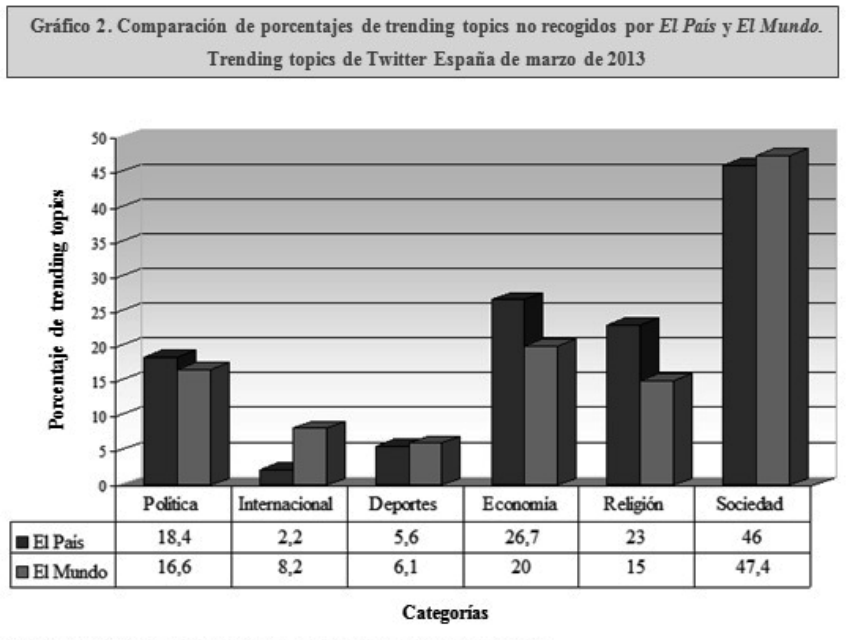

Fuente: Twitter, El País y El Mundo, marzo de 2013. Elaboración propia

Por otro lado, a pesar de que se trata de dos diarios distintos, ambos han protagonizado cifras muy similares de trending topics no reseñados en sus noticias. Tanto $E l$ País como El Mundo prácticamente coinciden en sus porcentajes de 'Política', 'Deportes' y 'Sociedad', en los que las diferencias no han alcanzado el $2 \%$.

Además, los contenidos de los temas sobre los que no ofrecieron información coinciden en su mayoría, lo que subraya que las agendas de ambos medios conciertan casi por completo en su registro de los temas de Twitter. Es importante recordar que el hecho de que estas agendas concuerden en este punto no significa que sean completamente coincidentes, porque para inferir una conclusión de esas características sería necesario analizar el contenido íntegro de cada uno de los diarios, y no sólo aquel relacionado con los trending topics de Twitter, como propone esta investigación.

Efectivamente los dos periódicos han dado cobertura a la mayoría de los temas que se encuentran tras los trending topics, sin embargo, es importante añadir que cerca del $80 \%$ de ellos han sido abordados en el mismo lapso temporal por los diarios y los usuarios, llegando incluso a ser reseñados antes por los tuiteros que por estos medios en algunas ocasiones.

Respecto a este punto, M. de Ramón` opina que "ahora mismo, gracias a las redes sociales, las fuentes se están convirtiendo en emisoras directas de noticias. Hasta ahora, si seguimos la tradición de la cadena de comunicación: fuente-periodistas-re-

9 Declaraciones de M. de Ramón en entrevista concedida a la autora el día 26 de junio de 2013. 
ceptores o público, los periodistas tenían un papel crucial porque eran los que recibían los 'premensajes' de las fuentes, los enviaban a los medios y los traducían a su lenguaje propio para difundirlo al público. Ahora las fuentes pueden puentear a los medios, lo que también hace peligrar el trabajo de los periodistas como intermediarios, que ahora tienen que $[\ldots]$ ponerse al servicio de las fuentes".

En los acontecimientos deportivos, por ejemplo, es relativamente sencillo que los internautas se conviertan en fuentes directas y publiquen informaciones prácticamente en el mismo momento en que se producen los hechos. No obstante, existen otros sucesos a los que el público no tiene este tipo de acceso. Son principalmente los asuntos relacionados con la política, la economía y el panorama internacional, de los que se obtienen informaciones que, primero, no son fáciles de conocer si no es a través de los medios o las fuentes primarias y, segundo, necesitan un nivel de interpretación mayor del que pueden ofrecer la mayoría de los usuarios de Twitter.

Los contenidos políticos han constituido uno de los patrones más claros en cuanto a que muestran claramente la dirección en la que fluye la información y la correlación que existe entre las agendas de los medios y el público.

El 17\% de los trending topics políticos fueron ignorados por El País y El Mundo. Esto significa que registraron un promedio de 179 de estos asuntos políticos, entre los que aproximadamente el $70 \%$ versó sobre corrupción en España y partidos políticos. Para ello, el diario de Prisa publicó un total de 414 noticias, mientras que el de Unidad Editorial logró sumar cuatro más.

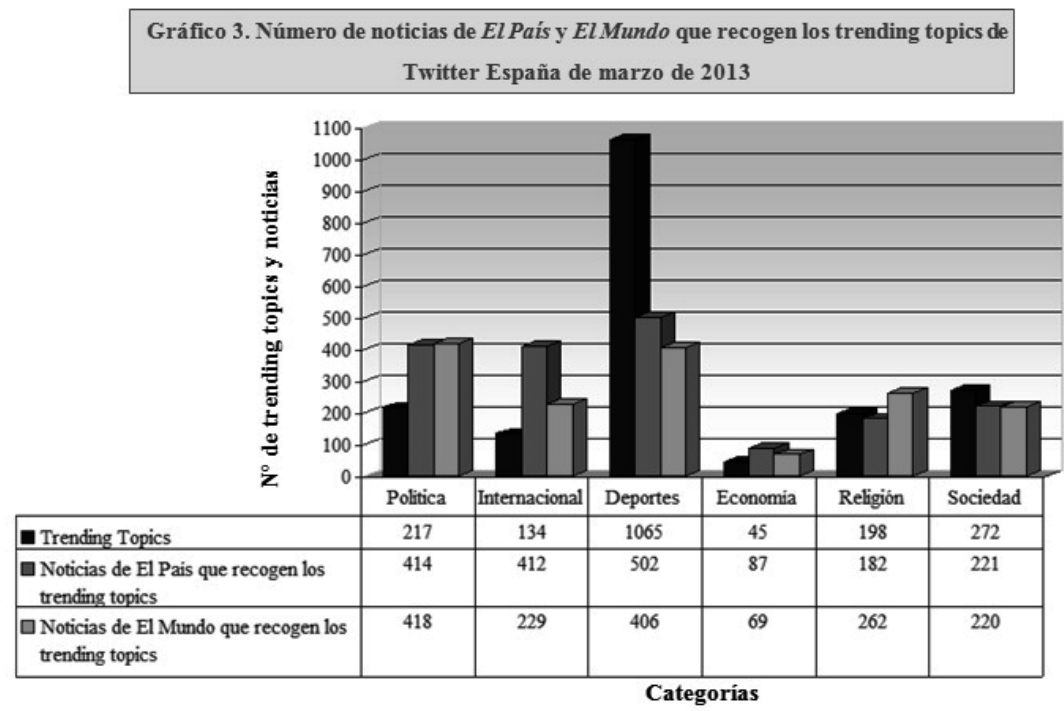

Fuente: Twitter, El Pais y El Mundo, marzo de 2013. Elaboración propia

No sólo esta cifra es muy similar en ambos diarios, sino que la temática de las noticias también coincide. Ambos han dado una importancia especial al seguimiento de los casos de corrupción política en España, como el caso Bárcenas y el caso Nóos, que El Mundo y El País se afanaron en seguir minuto a minuto. Los usuarios no tenían po- 
sibilidad de acceder de forma directa a determinadas informaciones y avances en estos casos por lo que tuvieron que esperar a que fueran recogidos por los medios para poder comentarlos y discutirlos en la red social.

En estas temáticas, a diferencia de lo que ocurría en eventos deportivos y religiosos, existe un claro lapso temporal entre el momento en que se produce el hecho (véase por ejemplo la declaración de Diego Torres en el juzgado) y el instante en que los usuarios de Twitter comienzan a comentar sobre él pues en medio de ambas acciones se encuentra la intermediación de los medios. Los internautas comentan en la red social aquello que observan en los medios de comunicación, sobre todo si se trata de temas complejos como los políticos o económicos, a los que no pueden acceder si no es a través de ellos.

La misma intermediación se observa cuando son los políticos los protagonistas de los trending topics y las informaciones. Detrás de aproximadamente el 65\% de los temas de moda centrados en la figura de un político español se encontraron las declaraciones de los propios políticos, que fueron recogidas por los medios.

Respecto a las informaciones sobre partidos, ambos diarios no registraron porcentajes similares de trending topics relacionados con estas organizaciones (18\% en $E l$ país y $21 \%$ en El Mundo), pero sobre aquellos que sí publicaron noticias, no lo hicieron en una proporción desmesurada. La cabecera de Prisa acumuló un total de 108 noticias sobre 27 temas de moda y la de Unidad Editorial 124 sobre 26.

Casi el 70\% de los temas de moda en Twitter sobre partidos fueron acaparados por el PP y el PSOE, y estos fueron, así mismo, los protagonistas de casi el $80 \%$ de las informaciones publicadas por nuestras cabeceras sobre partidos políticos. De la similitud de estas cifras se desprende que los usuarios de Twitter otorgan a estos temas una importancia semejante a la conferida por los medios.

En general, se puede concluir que los temas políticos son comentados por los internautas a partir de las informaciones de los medios porque se trata de asuntos actuales y complejos, tanto en su acceso como en su análisis.

Este liderazgo de los medios se vuelve incluso más esencial en los asuntos internacionales, ya que las distancias espaciales complican el acceso directo a la información.

Respecto a este tipo de temas que se enmarcan en el plano internacional, cabe destacar que, en general, los usuarios de Twitter los han abordado en menor proporción que nuestros dos diarios digitales. Atendiendo a los asuntos europeos, por ejemplo, los internautas acumularon un total de 55 temas de moda, de los que El País sólo ignoró dos y El Mundo tres; el resto fueron recogidos en 229 y 102 noticias respectivamente. La diferencia entre uno y otro es bastante elevada, lo que hace pensar que la cabecera de Prisa ofrece mayor cantidad de información en su sección internacional, aunque esta aborda los mismos temas que su análogo.

De esas noticias publicadas, 132 y 74 correspondientemente fueron destinadas a la situación económica y el rescate de Chipre, de la misma forma que los propios usuarios dedicaron la mayoría de los temas de moda de la Unión Europea a la misma causa, y lo hicieron siguiendo una línea cronológica marcada por las informaciones difundidas por los medios de comunicación. 
Lo mismo ocurrió con otros asuntos internacionales como la muerte del presidente venezolano Hugo Chávez el cinco de marzo de 2013. Aunque la noticia inicial del fallecimiento pudo ser conocida por los internautas gracias a medios de otros países, el seguimiento posterior muestra que los usuarios se informaron a través de los medios españoles y que otorgaron a las informaciones una importancia similar a la conferida por los medios, que llegaron a publicar 182 noticias sobre el tema.

Los ejemplos de Chipre y Venezuela, sumados a los resultados obtenidos en otras cuestiones como la guerra entre Corea del Norte y Corea del Sur, muestran que en materia internacional los usuarios de Twitter dependen mucho más de la información ofrecida por los medios. La razón es que la distancia geográfica y muchas veces lingüística genera a los tuiteros la imposibilidad de acercarse al acontecimiento directamente, haciendo necesaria la intermediación de los medios como acceso y filtro de la información.

Esta situación no se produce, sin embargo, en la mayoría de los temas relacionados con el mundo deportivo. Los diarios digitales no constituyen un referente informativo en eventos deportivos ya que la mayor parte de ellos son seguidos en directo por los espectadores, que tienen acceso a ellos principalmente a través de la radio o la televisión. Estos medios sí ofrecen la información en tiempo real, lo que provoca que algunos internautas publiquen en sus cuentas de Twitter comentarios sobre los eventos utilizando hashtags comunes que permiten a otros usuarios seguirlo vía Twitter.

Nuestros diarios de referencia, por el contrario, no acostumbran a dar una cobertura en tiempo real de los eventos deportivos, salvo que estos sean de mucha relevancia nacional. A pesar de esta limitación de los diarios digitales en cuestiones deportivas, no podemos olvidar que la intermediación mediática sigue estando ahí en el sentido de que no todas las personas pueden ver los eventos en vivo, por lo que utilizan los medios para seguir en directo lo que ocurre.

En lo que respecta a las cuestiones religiosas, hay que destacar que El Mundo ha recogido con más frecuencia estos asuntos. Este diario publicó informaciones sobre un $8 \%$ más de trending topics que El País, que no recogió un $23 \%$ de ellos.

En estos temas se observa una situación anómala en la que se concentran tanto características identificadas en la categoría de internacional como otras definidas para las temáticas deportivas. En materia religiosa los temas de moda se han dividido en dos bloques: el Cónclave para elegir al nuevo Papa y la Semana Santa, y cada uno de ellos ha manifestado unas características determinadas en función de su naturaleza. El primero es un evento de repercusión internacional y gran resonancia mediática al que los tuiteros han tenido acceso actualizado gracias a las informaciones de los medios. Ambos diarios han recogido el $100 \%$ de los 95 temas de moda comentados por los usuarios, y lo han hecho en 127 noticias en el caso de El País y en 114 en el de El Mundo.

El segundo se enmarca dentro del tipo de aconciminetos que los usuarios acostumbran a seguir y tuitear en directo. El seguimiento de los eventos de la Semana Santa ha sido mayor en la red social que en los medios pues mientras que los usuarios consiguieron 103 temas de moda nacionales, El País y El Mundo dejaron fuera de sus publicaciones el $44 \%$ y $29 \%$ de ellos respectivamente. Esto que hace pensar que en este caso, los dos diarios no han marcado los temas de la agenda del público. 
Para concluir, sólo resta exponer el caso de dos temáticas que han seguido patrones similares en cuanto a la correlación de su seguimiento en Twitter y en nuestros dos diarios: economía y sociedad.

Ambas han sido las dos categorías en las que se ha observado la relación más débil entre lo publicado por los tuiteros y lo recogido por El País y El Mundo. Por un lado, la cabecera de Prisa ha dejado fuera de su cobertura el $26.7 \%$ de las cuestiones económicas comentadas en Twitter y el $46 \%$ de las enmarcadas en 'Sociedad', y por otro, la de Unidad Editorial ha presentado resultados parecidos fijados en $20 \%$ y $47.4 \%$, respectivamente.

Es importante tener en cuenta que el hecho de que porcentajes tan altos no hayan sido reseñados por los diarios no significa que estos no publiquen informaciones sobre el mundo económico o sucesos. Lo que ocurre es que estos no han otorgado en su agenda el mismo nivel de importancia a los mismos temas que los usuarios.

En materia económica el mayor número de cuestiones ignoradas por ambas cabeceras se corresponde con aquellas centradas en empresas, pero respecto al resto, la agenda de los dos diarios sí ha concordado con la del público que, en cuestiones como las cotizaciones en bolsa y la situación de la banca y economía española, han acudido a los medios en busca de análisis e interpretación antes de comentar en Twitter.

Por su parte, en los temas de moda enmarcados dentro de 'Sociedad', destacamos que, a pesar de que han sido los menos recogidos por nuestros dos diarios, los que sí registraron ocuparon bastante espacio en las noticias, y además lo hicieron en ambas cabeceras de manera muy similar. El País publicó en total 221 piezas informativas sobre los 146 temas de moda que recogió, y El Mundo publicó 220 para 143 temas.

Es interesante subrayar el caso de las manifestaciones. El País y El Mundo registraron la mayoría de ellas, pero no fueron los primeros en ofrecer la información de lo que estaba ocurriendo. Utilizando los hashtags propuestos para estas protestas, los manifestantes narraron vía Twitter lo que estaba pasando, mientras que los medios no lo publicaron en ese mismo momento. Sin embargo, cuando lo hicieron, incluyeron en sus noticias el análisis y el contexto sobre los que comentaron los internautas posteriormente.

Es en este punto donde mejor se refleja el papel de los medios como intermediarios entre la información, a la que no todos los usuarios tienen acceso, y el público; y donde se observa la correlación que existe entre ambas agendas.

\section{Conclusiones}

A la luz de los resultados obtenidos en el análisis de los datos presentados, se han extraído algunas conclusiones.

La primera es que la opinión pública reflejada en la red social Twitter ha demostrado componer su agenda temática en torno a dos líneas distintas. Ha quedado probado que los usuarios están interesados tanto en la realidad más actual, aquella que suelen recoger los medios de comunicación, como en otro tipo de cuestiones más ligadas a la propia naturaleza de Twitter y al entretenimiento. En este último sentido se ha advertido una predisposición especial de los internautas a participar en temas colectivos con el objetivo de otorgarse visibilidad a sí mismos y de interactuar con usuarios de intereses y opiniones similares, sobre todo en lo que a música se refiere. 
También se ha observado una creciente tendencia a relacionarse con los medios a través de sus contenidos y a utilizar esta red social como un complemento de otras actividades que realizan de forma simultánea, como por ejemplo, el consumo de contenidos televisivos o radiofónicos.

Por otro lado, dentro de los asuntos ligados a la información de actualidad, podemos destacar que los internautas han otorgado a las cuestiones relativas al mundo del deporte mayor importancia que al resto. Los asuntos deportivos han ocupado casi el $30 \%$ de la agenda pública, lo que evidencia un interés de los usuarios por todo tipo de deportes, entre los cuales ha predominado el fútbol. Los tuiteros también han expuesto cierta predilección por las cuestiones políticas y sociales, mientras que aquellas relacionadas con la economía y el plano internacional han ocupado muy poco espacio en su agenda.

La segunda conclusión que se desprende de esta investigación es que existe una clara correspondencia entre la agenda del público y la establecida por los medios de comunicación tradicionales. Dejando al margen la parte de la agenda pública referida a los temas originarios de Twitter, hemos observado que en lo que respecta a las temáticas más informativas, estas han encontrado su reflejo en los medios analizados, es decir, que los asuntos comentados por los usuarios de Twitter en la red social han sido en su mayoría abordados también por los medios.

En este punto, uno de los aspectos importantes radica en que esta correlación de agendas no siempre depende de la influencia de los medios en la opinión pública, sino que en algunas ocasiones, la similitud de ambas se debe a que medios y usuarios tienen un acceso semejante a la información. Algunos de los ejemplos han demostrado que a veces los internautas publican información no basándose en lo emitido en los medios, sino en su propia experiencia y conocimiento.

Estas han sido, sin embargo, excepciones concentradas en torno a algunos de los asuntos deportivos, religiosos y eventos concretos, pues en general han sido los medios los que han marcado con anterioridad no sólo las temáticas sobre las que posteriormente discutiría la opinión pública en Twitter, sino el propio espacio e importancia otorgados a cada una de ellas. De esta forma, algunas cuestiones como los casos de corrupción en España, la muerte de Hugo Chávez, el cónclave para elegir nuevo Papa o el rescate de Chipre han protagonizado un interés mediático que los usuarios han recogido en Twitter en una proporción muy similar.

Estos ejemplos resaltan otra observación importante y es que el nivel de influencia de los medios sobre la agenda pública depende y varía en función de los temas. Mientras que se ha observado una fuerte influencia mediática en lo que respecta a asuntos políticos e internacionales, en otros como los tecnológicos o científicos ha sido mucho menor, lo que evidencia la imposibilidad de extraer conclusiones generales sobre la perfecta correlación de la agenda pública y mediática, y sobre el poder de esta última de marcar los temas del debate público. Somos, por tanto, conscientes de que las aquí referidas no son ideas universales ni irrefutables, principalmente porque se desprenden de un análisis sujeto a un periodo temporal cerrado. Sin embargo, consideramos que este trabajo ha demostrado su validez y justificación en varios aspectos que conviene tener en cuenta. 
En primer lugar, pensamos que puede constituirse como una aproximación metodológica al análisis del entorno digital de Twitter, un terreno cuyo valor empírico ha empezado recientemente a despertar el interés de los investigadores en el plano de la comunicación. Las herramientas utilizadas para su elaboración, lejos de aproximarse a los métodos tradicionales, destacan por su novedad y su facilidad de uso, así como por la precisión de los datos aportados y su cualidad de adaptarse a los objetivos de cada estudio. Estas posibilidades de los nuevos métodos de análisis, sumadas a la garantía de la veracidad y validez de los resultados obtenidos, convierten estas herramientas en instrumentos válidos que pueden ser considerados útiles en el desarrollo de futuras investigaciones que se enmarquen dentro de la red social Twitter.

En segundo lugar, consideramos oportuno atender a que, de la misma forma que esta investigación se ha centrado en la parte de la agenda pública compuesta por los temas de actualidad, aquellas líneas temáticas más relacionadas con el entretenimiento y la participación constituyen otra vía de análisis que, si bien no es excesivamente relevante en el plano de la comunicación mediática, sí puede llegar a serlo en comunicación publicitaria, en la medición de audiencias o incluso en estudios sociológicos que se centren en las características de la unión comunitaria de los usuarios.

\section{Referencias bibliográficas}

BERELSON, Bernard (1952): Content analysis in communication research. New York, Hafner.

BOUZA, Fermín y RODRÍGUEZ, Raquel (2004): “Examinando la Agenda Pública en España, la Agenda Personal y el Arrea de Impacto de la Comunicación Política entre el 11 y el 14 de Marzo de 2004". Ponencia presentada en The 55th Annual Conference of International Communication Association. New York, USA, 2005, May 26-30: http://pendientedemigracion.ucm.es/info/socvi/BOUZA/NUEVA1 /Textos/050607/espica.pdf [fecha de consulta: 17 de marzo de 2013].

BRUDER, Katherine (2008): "Agenda-Setting of International News in the United States and Its Decline by Using New Technologies". Communication Senior Seminar, 14 de abril, California, Alma College: http://www.katherinebruder.com/Writing_\&_Design_files/Agenda\%20Setting.pdf [fecha de consulta: 15 de abril de 2013].

KRANE, Meghan (2010): The socially filtered media agenda: a study of Agenda-Setting among news outlets on Twitter. Columbia, University of Missouri.

KRIPPENDORFF, Klaus (1990): Metodología de análisis de contenido: teoría y práctica. Barcelona, Paidós.

KVALE, Steinar (2011): Las entrevistas en investigación cualitativa. Madrid, Morata.

LIPPMANN, Walter (1922): Public Opinion. New York, Macmillan.

MCCOMBS, Maxwell y SHAW, Donald (1972): “The Agenda'Setting Function of Mass Media". The Public Opinion Quarterly, no 36. Oxford, Oxford University Press, pp. 176-187. 
MCCOMBS, Maxwell (2005): "Look at Agenda-Setting: past, present and future". Journalism Studies, vol. 6, no 4. London, Routledge, pp. 543-557.

MCCOMBS, Maxwell (2006): Estableciendo la agenda: el impacto de los medios en la opinión pública y en el conocimiento. Barcelona, Paidos Ibérica.

MERAZ, Sharon (2009): "Is There an Elite Hold? Traditional Media to Social Media Agenda-Setting Influence in Blog Networks". Journal of Computer-Mediated Communication, no 14, 682-707. http://onlinelibrary.wiley.com/doi/10.1111/j.10836101.2009.01458.x/pdf [fecha de consulta: 10 de mayo de 2013].

ORIHUELA, José Luis (2008): "Internet: la hora de las redes sociales". Nueva Revista de politica, cultura y arte, no 119, pp. 57-62: https://docs.google.com/file/d /0B7KQbnZ7HHLQN2U5ZDU1YWEtZDNmNi00YjI0LThmMzAtMWI4YTEw N2Y3MWE1/edit?hl=es\&pli=1 [fecha de consulta: 20 de febrero de 2013].

PEW RESEARCH CENTER'S PROJECT FOR EXCELLENCE IN JOURNALISM (2010): "New Media, Old Media: How Blogs and Social Media Agendas Relates and Differ from Traditional Press". Washington, DC.: http://www.journalism.org /analysis_report/new_media_old_media [fecha de consulta: 15 de junio de 2013].

PEW RESEARCH CENTER'S PROJECT FOR EXCELLENCE IN JOURNALISM (2011): "How Mainstream Media Outlets Use Twitter. Content Analysis Shows an Evolving Relationship". Washington, DC.: http://www.journalism.org/node/27311 [fecha de consulta: 15 de junio de 2013].

RODRÍGUEZ, Raquel (2004): Teoría de la agenda-setting: aplicación a la enseñanza universitaria. Alicante, Observatorio Europeo de Tendencias Sociales.

ROSTOVTSEVA, Nataliya, (2009): "Inter-Media Agenda Setting role of the blogosphere: A content analysis of the Reuters photo controversy coverage during the Israel-Lebanon conflict in 2006". Master Dissertation, Chapel Hill, University of North Carolina, School of Journalism and Mass Communication: http://edoc.bibliothek.unihalle.de/servlets/MCRFileNodeServlet/HALCoRe_derivate_00005325/Rostovtseva_Intermedia.pdf [fecha de consulta: 13 de abril de 2013].

RUIZ OLABUÉNAGA, José Ignacio (2012): Metodología de la investigación cualitativa. Bilbao, Universidad de Deusto.

TAYLOR, Steven y BOGDAN, Robert (1984): Introducción a los métodos cualitativos de investigación. Barcelona, Paidós.

WIMMER, Roger D. \& DOMINICK, Joseph R. (2006): Mass Media Research: an introduction. USA, Thomson Wadsworth. 